\title{
Improving Growth, Fruit Set, Yield, Fruit Quality and Shelf Life of "Costata" Persimmon
}

\author{
A. Abd El-Baree*, Magda M. Nasr ${ }^{* *}$ and M.A. Fathi* ${ }^{* *}$ \\ *Paramon Research Station and ${ }^{* *}$ Horticulture Research \\ Institute, Agricultural Research Centre, Cairo, Egypt.
}

\begin{abstract}
TIS investigation was conducted at Hort. Res. Station Orchard, Paramon, Dakahlia governorate during 2010 and 2011 seasons to study the effect of foliar application of Milagrow $(250 \mathrm{gm} / 5 \mathrm{l})$, Pepton, $\mathrm{GA}_{3}$ (single or in combinations) and Folgers on vegetative growth, leaf content of minerals and chlorophyll, fruit set, yield and fruit quality as well as shelf life of "Costata" persimmon trees (12 years old) grafted on seedling rootstock on loamy soil.

The present results showed that, all treatments improved all growth and yield parameters compared to the control. With regard to the yield and fruit quality, the most effective treatment was spraying "Costata" persimmon trees three times (at full bloom, fruit set and June fruit drop) with a combined treatment of Peptone at $1000 \mathrm{ppm}+$ $\mathrm{GA}_{3}$ at $20 \mathrm{ppm}$ or Milagrow at $50000 \mathrm{ppm}+$ Pepton at $1000 \mathrm{ppm}+$ $\mathrm{GA}_{3}$ at $20 \mathrm{ppm}$.
\end{abstract}

Keywords: Milagrow, Pepton, Folgers and GA3.

Japanese persimmon (Diospyros kaki L.) is one of deciduous fruit trees which belong to the family Ebenaceae. It has been introduced to Egypt in 1911 (Baghdady and Mineasy, 1964), but the main problem is the high fruit drop before maturity, subsequently the final yield is not economical. However, improving yield and fruit quality without adversely effect on the environment is a major goal of horticulturists and could be achieved by using bio-stimulants which may contain microorganisms or natural products as cytokinins, amino acids and organic acids (Russo and Berlyn, 1990). Abd El-Ghany (2005) reported that, fruits June drop occurred due to the competition among the fruits on the nutrients, water and the defect in hormonal balance and could improved vegetative growth, yield and fruit quality of "Costata" persimmon by foliar application of some bio-stimulants and $\mathrm{GA}_{3}$ which affect the fruit shelf life (Awad and Amenomori, 1971). Looney (1993) found that early fruit cell division normally influenced by natural growth hormones especially cytokinin. $\mathrm{GA}_{3}$ significantly increased persimmon fruit set, firmness, colour and T.S.S. (ElFakharani et al., 1990 and Hasegawa et al., 1991), while it did not affect tannins or fruit juice acidity (Mokhtar and Wally, 1999). Moreover, Guirguis et al. (2009) got the highest fruit set and yield as well as the lowest fruit drop of "Mackawa Jiro" persimmon by $\mathrm{GA}_{3}$ at10 ppm + Sitofex at $5 \mathrm{ppm}$ at full bloom. Also, $\mathrm{GA}_{3}$ at $50 \mathrm{ppm}$ at full bloom improved pear yield and fruit characters (Nasr et al., 2009). 
Folgers bio-stimulant has a positive effect on pear foliage, leaf chlorophyll content, fruit set, yield and fruit characters (Eissa et al., 2007). Also, Milagrow (Brassinosteroids, BRs) stimulated cell division and elongation, flower bud differentiation, carbohydrate assimilation and ATP activity subsequently improved vegetative growth, enhanced physiological status and directed trees to earlier harvest as well as increased fruit yield and quality (Clause, 1996, Wang et al., 2004, Mussig, 2005, Gomes et al., 2006, Symons et al., 2006 and Gabr et al., 2011).

This study aims to increase fruit set, yield, fruit characters and fruit shelf life as well as decrease fruit drop through studying the effect of spraying ( Milagrow, Pepton and $\mathrm{GA}_{3}$ ) alone or combined and folgers at full bloom, fruit set and at fruit drop of " Costata" persimmon.

\section{Materials and Methods}

The present study was performed at Hort. Res. Station, Paramon, Dakahlia governorate, Egypt during 2010 and 2011 seasons. We used 12-year-old "Costata" persimmon trees budded on seedling rootstock and grown on loamy soil. Twenty seven trees (3trees / treatment) were nearly uniform, planted at $4 \times 5$ $\mathrm{m}$ apart and received similar cultural practices. The chosen trees were spayed three times: at full bloom, fruit set and at June fruit drop with:

- $\mathrm{GA}_{3}$ at $20 \mathrm{ppm}$.

- Milagrow at $50000 \mathrm{ppm}$ (contains: 20\%P, $10 \% \mathrm{~K}, 3 \% \mathrm{~B}$ and $0.2 \%$ Brassinosteroids).

- Pepton at $1000 \mathrm{ppm}$ (based on the energizing power of free amino acids) produced by A.P.C. Europ Co., Avsan Julain, Spain.

- Folgers at $1000 \mathrm{ppm}$ (contains unidentified natural growth stimulants).

- $\mathrm{GA}_{3}+$ Pepton.

- $\mathrm{GA}_{3}+$ Milagrow.

- Milagrow + Pepton.

- Milagrow + Pepton $+\mathrm{GA}_{3}$.

- Control.

Five branches per tree were labeled and the treatments were arranged in a complete randomized block design to determine: shoot length, number of leaves/ shoot and leaf area (Bleasdale, 1978). Samples of twenty leaves / tree were taken in late September of the $2^{\text {nd }}$ season to determine mineral content $(\mathrm{N}, \mathrm{P}$, and $\mathrm{K})$ (Myers and Ferree, 1983). Leaf chlorophyll content was measured as SPAD by chlorophyll meter (Minolta Corporation, Ramsey NJ, USA). Fruit set was assessed as well as number of fruit / tree and fruit yield. These data were used to estimate crop monetary value considering a farm-gate price of LE $1.5 / \mathrm{kg}$ for fruit weighed $<90 \mathrm{~g}$, LE $2.0 / \mathrm{kg}$ for fruit weighed $90-110 \mathrm{~g}$ and LE $2.5 / \mathrm{kg}$ for fruit weighed $>110 \mathrm{~g}$. Picking date ( at the stage of greenish yellow colour) was according to Wally et al. (1999). Sample of 10 mature fruits / tree were randomly selected to assess skin colour, fruit weight, size and dimensions. Skin firmness was measured by hand pressure tester (MEG, Co.). Thirty fruits were

Egypt. J. Hort. Vol. 40, No.2 (2013) 
stored at room temperature (at $20^{\circ} \mathrm{c}$ ) or cold storage (at $5^{\circ} \mathrm{c}$ and $80-85 \% \mathrm{RH}$ ) to assess their shelf life. Fruit juice was used to estimate T.S.S., acidity and Tannins (A.O.A.C., 1970).

Data were statistically analyzed according to Snedecor and Cochran (1990). L.S.D. test was used for comparison between means. Also, regression equations were assessed between leaf area and fruit set percentage, fruit yield and juice TSS as well as correlation coefficient.

\section{Results and Discussion}

\section{Vegetative Growth}

The response of vegetative growth (shoot length and diameter as well as number of leaves and leaf area) is illustrated in Table 1. The present results showed that all treatments had positiveeffect on vegetative growth compared to control. Also, data revealed that Folgers $(92.67$ and $84.67 \mathrm{~cm}$ ) and Peptone $(90.0$ and $73.67 \mathrm{~cm})$ induced the longest significant shoots compared to the control $(33.33$ and $47.33 \mathrm{~cm}$ ) during the two seasons, respectively. However, the same trend was noticed with shoot diameter, number of leaves and leaf area of "Costata" persimmon. Unexpectedly, all mixed treatments induced lower vegetative growth than individual ones. This phenomenon may point out an antagonistic effect of the present combinations.

TABLE 1. Effect of stimulant treatments on vegetative growth of "Costata" Persimmon trees.

\begin{tabular}{|l|c|c|c|c|c|c|c|c|}
\hline \multirow{2}{*}{ Treatments } & \multicolumn{2}{|c|}{$\begin{array}{c}\text { Shoot Length } \\
(\mathbf{c m})\end{array}$} & $\begin{array}{c}\text { Shoot diameter } \\
(\mathbf{c m})\end{array}$ & \multicolumn{2}{c|}{$\begin{array}{c}\text { Number of } \\
\text { leaves }\end{array}$} & \multicolumn{3}{c|}{$\begin{array}{c}\text { Leaf area } \\
\left(\mathbf{c m}^{2}\right)\end{array}$} \\
\cline { 2 - 9 } & $\mathbf{2 0 1 0}$ & $\mathbf{2 0 1 1}$ & $\mathbf{2 0 1 0}$ & $\mathbf{2 0 1 1}$ & $\mathbf{2 0 1 0}$ & $\mathbf{2 0 1 1}$ & $\mathbf{2 0 1 0}$ & $\mathbf{2 0 1 1}$ \\
\hline Control & 33.33 & 47.33 & 0.683 & 0.650 & 13.00 & 19.67 & 23.74 & 25.31 \\
\hline $\begin{array}{l}\text { Milagrow } \\
\text { (50000 ppm) }\end{array}$ & 83.67 & 85.67 & 0.823 & 1.020 & 18.33 & 20.67 & 29.64 & 29.11 \\
\hline Pepton (1000 ppm) & 90.00 & 73.67 & 0.906 & 0.910 & 26.00 & 25.67 & 34.52 & 34.29 \\
\hline Folgers (1000 ppm) & 92.67 & 84.67 & 0.930 & 1.120 & 27.33 & 25.00 & 36.55 & 35.91 \\
\hline $\begin{array}{l}\text { Gibberellins } \\
(\text { (20 ppm) }\end{array}$ & 82.33 & 100.3 & 0.760 & 1.000 & 20.67 & 24.67 & 36.95 & 38.51 \\
\hline Milagrow + Pepton & 62.00 & 58.00 & 0.800 & 0.760 & 18.00 & 19.67 & 27.40 & 28.78 \\
\hline $\begin{array}{l}\text { Milagrow + } \\
\text { Gibberellins }\end{array}$ & 52.67 & 52.33 & 0.766 & 0.800 & 19.33 & 22.33 & 30.03 & 31.30 \\
\hline $\begin{array}{l}\text { Pepton + } \\
\text { Gibberellins }\end{array}$ & 60.67 & 62.67 & 0.800 & 0.720 & 19.33 & 20.33 & 26.73 & 26.69 \\
\hline $\begin{array}{l}\text { Milagrow + Pepton+ } \\
\text { Gibberellins }\end{array}$ & 57.33 & 76.00 & 0.763 & 0.886 & 19.00 & 21.67 & 28.01 & 27.42 \\
\hline L.S.D at 0.05 & 0.93 & 0.92 & 0.181 & 0.181 & 1.06 & 0.91 & 1.62 & 1.94 \\
\hline
\end{tabular}


However, these results are in harmony with those of Kauschmann et al. (1996) who stated that Milagrow (Brassinosteroids, BR) play an important role in the control of cell division and elongation, while Mussig (2005) showed that BR apparently coordinates and integrates diverse process required for growth, partly via interactions with phytohormones. Moreover, Gabr et al. (2011) noticed a progressive increment of apricot leaf growth parallel to BR concentrations in spraying solution.

However, Walter and Nawacki (1978) showed that amino acids can affect plant growth and development through their influence on $\mathrm{GA}_{3}$ biosynthesis, while Thon et al. (1981) stated that amino acids provide plant cells an available source of $\mathrm{N}$ which can be taken more rapidly than organic nitrogen. Meanwhile, Kowalczyk and Zielony (2008) found that amino acids treatment have positive effect on plant growth where they significantly mitigate the injuries caused by a biotic stresses, while Shehata et al. (2011) said that amino acids play an important role in plant metabolism and protein assimilation as well as may take part in micronutrients absorption and serve as a source of nitrogen (Datir et al., 2012). Also, Eissa et al., (2007) found a strong positive effect of Folgers treatments on pear foliage characters. However, $\mathrm{GA}_{3}$ treatments significantly increased vegetative growth of Araucaria heterophylla seedlings (Gul et al., 2006), "Canino" apricot (Hoseen et al., 2008) and "Leconte" pear (Aly et al., 2012) and Pumpkin (Shirzad et al. 2013).

\section{Leaf chlorophyll and nutrient content \\ Chlorophyll}

Results in Table 2 showed insignificant effect between the present treatments and the control (43.93 and 45.25 SPAD), except Pepton treatment in the first season (49.73 SPAD). Otherwise, Folgers treatment induced the lowest leaf chlorophyll content through the two studied seasons (42.82 and 43.12 SPAD). However the former investigation showed that Milagrow treatment greatly stimulated accumulation of photosynthates (Krizek and Mandava, 1983) as well as they were essential for many physiological functions (Montoya et al., 2005) where they induced progressive increment of apricot leaf chlorophyll (Gabr et al., 2011). Moreover, Abd El-Aziz and Balbaa (2007) and Shehata et al. (2011) reported that amino acids spray caused an increase in photosynthetic pigments content. Meanwhile, leaf chlorophyll content was significantly increased by the treatment of Folgers (Eissa et al., 2007), Pepton (Ibrahim et. al., 2010) and $\mathrm{GA}_{3}$ (Gross et al., 1984 and Shahin et al. 2010).

\section{Leaf nutrient content}

Data in Table 2 showed that $\mathrm{GA}_{3}$ treatment significantly induced the highest nitrogen leaf content (1.67\%) followed by Milagrow + Pepton (1.46\%) Milagrow $+\mathrm{GA}_{3}(1.36 \%)$ and Milagrow + Pepton $+\mathrm{GA}_{3}(1.35 \%)$, respectively. With respect to phosphorus leaf content, data showed unclear trend where $\mathrm{P}$ leaf content of control, $\mathrm{GA}_{3}$ and Millagrow $+\mathrm{GA}_{3}$ treatments were the same, while the rest treatments induced less $\mathrm{P}$ leaf content. However, Folgers $(1.41 \%)$ and Milagrow + Pepton (1.34\%) treatments significantly induced higher K leaf 
content than both the control $(1.16 \%)$ and the rest treatments. Generally, the obtained results are in agreement with those of Eissa et el. (2007) and Aly et al. (2012) who found no clear trend to spray of Folgers and $\mathrm{GA}_{3}$ on pear leaf content of NPK. Otherwise, Abo Sedera et al. (2010) on strawberry and Shahin et al. (2010) on " Anna " apple reported higher NPK leaf content due to Pepton (amino acids), Fertifol (25\% N, 16\% P, 12\%K, 0.25\% Mg, 300 ppm Zn, 1900 ppm Mn. $850 \mathrm{ppm} \mathrm{Cu}, 100 \mathrm{ppm}$ Mo and $200 \mathrm{ppm} \mathrm{B}$ ) and $\mathrm{GA}_{3}$ at $20 \mathrm{ppm}$. Also, Hassan et al. (2010) on "Hollywood" plum trees found that $\mathrm{GA}_{3}$ and Aminofert (20\% amino acids, $12 \%$ organic acids and $3.6 \%$ chelated micro-elements) caused a pronounced increase in leaf $\mathrm{N}$ and $\mathrm{K}$ content while leaf $\mathrm{P}$ content decreased in both seasons of study.

TABLE 2. Effect of stimulant treatments on leaf chemical content of "Costata" Persimmon trees.

\begin{tabular}{|l|r|r|r|r|c|}
\hline \multirow{2}{*}{ Treatments } & \multicolumn{2}{|c|}{$\begin{array}{c}\text { Chlorophyll } \\
\text { (SPAD reading) }\end{array}$} & N (\%) & P (\%) & K (\%) \\
\cline { 2 - 6 } & $\mathbf{2 0 1 0}$ & $\mathbf{2 0 1 1}$ & $\mathbf{2 0 1 1}$ & $\mathbf{2 0 1 1}$ & $\mathbf{2 0 1 1}$ \\
\hline Control & 43.93 & 45.25 & 1.000 & 0.140 & 1.160 \\
\hline Milagrow (50000 ppm) & 44.91 & 49.11 & 1.250 & 0.120 & 1.090 \\
\hline Pepton (1000 ppm) & 49.73 & 48.74 & 1.110 & 0.120 & 1.070 \\
\hline Folgers (1000 ppm) & 42.82 & 43.12 & 0.940 & 0.110 & 1.410 \\
\hline Gibberellins (20 ppm) & 44.14 & 43.15 & 1.670 & 0.140 & 1.020 \\
\hline Milagrow + Pepton & 46.38 & 47.56 & 1.460 & 0.130 & 1.340 \\
\hline Milagrow + Gibberellins & 46.35 & 44.09 & 1.360 & 0.140 & 1.270 \\
\hline Pepton + Gibberellins & 44.81 & 46.48 & 1.180 & 0.110 & 1.090 \\
\hline $\begin{array}{l}\text { Milagrow + Pepton + } \\
\text { Gibberellins }\end{array}$ & 45.15 & 47.49 & 1.350 & 0.120 & 1.220 \\
\hline L.S.D at 0.05 & 3.78 & 4.07 & 0.245 & 0.001 & 0.155 \\
\hline
\end{tabular}

Fruit set, number of fruits/tree, yield/tree and per Feddan

Results in Table 3 showed that, all treatments increased fruit set percentage compared to the control (30.6 and 32.67\%) However, $\mathrm{GA}_{3}$, Folgers and Milageow $+\mathrm{GA}_{3}$ induced the highest fruit set in the $1^{\text {st }}$ season $(58.69,54.40$ and $53.93 \%$ ) while in the $2^{\text {nd }}$, Pepton $+\mathrm{GA}_{3}, \mathrm{GA}_{3}$, Folgers and Milageow $+\mathrm{GA}_{3}$ were the highest $(62.20,58.33,57.46$ and $55.65 \%)$, respectively.

Number of fruits/tree significantly increased as a result of all treatments. However, in 2010 season Milagrow + Pepton, Pepton $+\mathrm{GA}_{3}$ and Pepton $+\mathrm{GA}_{3}$ + Milagrow get the highest number of fruits (250.0, 243 and 224.5), while in 2011 season Folgers, Pepton $+\mathrm{GA}_{3}$,pepton $\mathrm{GA}_{3}+$ Milagrow and Milagrow + Pepton were the highest respectively $(350.5,333.0,304,302.7$ and 301.5) compared to control (135 and 198.5). Moreover, the fruit yield per tree and per feddan significantly increased by all the present treatments compared to control (11.16 and $14.49 \mathrm{~kg} /$ tree as well as 2.230 and 2.893 ton / feddan). However, 
Pepton $+\mathrm{GA}_{3}$ gave the highest yield per tree $(29.72$ and $40.51 \mathrm{~kg})$ and per Feddan (5.940 and 8.097 ton) in the two studied seasons, respectively followed by Pepton $+\mathrm{GA}_{3}+$ Milagrow, and Milagrow + Pepton in the first season $(27.62$ and $26.72 \mathrm{~kg} /$ tree as well as 5.520 and 5.337 ton / feddan), respectively but in the second season followed Folgers, $\mathrm{GA}_{3}$ and Milagrow + Pepton (37.99, 36.48 and $32.68 \mathrm{~kg}$ and 7.660, 7.293 and 6.533 ton / Feddan ), respectively.

Table 3 showed the monetary value of " Costata " persimmon yield/tree as affected by the present treatments. It can be noticed that all treatments markedly increased yield monetary value than the control (16.75 and 21.73 LE). However, the most effective treatments were Pepton $+\mathrm{GA}_{3}(74.31$ and 101.30 LE) in the two studied seasons, respectively and $\mathrm{GA}_{3}$ treatment in $2^{\text {nd }}$ season only (91.19 LE).

TABLE 3. Effect of stimulant treatments on fruit characteristics of "Costata" Persimmon trees.

\begin{tabular}{|l|c|c|c|c|c|c|c|c|c|c|}
\hline \multirow{2}{*}{ Treatments } & \multicolumn{2}{|c|}{$\begin{array}{c}\text { Fruit set } \\
(\%)\end{array}$} & \multicolumn{2}{c|}{$\begin{array}{c}\text { Fruit } \\
\text { number / } \\
\text { Tree }\end{array}$} & \multicolumn{2}{c|}{$\begin{array}{c}\text { Yield / tree } \\
\text { (kg) }\end{array}$} & \multicolumn{2}{c|}{$\begin{array}{c}\text { Yield / } \\
\text { feddan } \\
\text { (Ton) }\end{array}$} & \multicolumn{2}{c|}{$\begin{array}{c}\text { Monetary } \\
\text { value / tree } \\
\text { (LE) }\end{array}$} \\
\cline { 2 - 11 } & $\mathbf{2 0 1 0}$ & $\mathbf{2 0 1 1}$ & $\mathbf{2 0 1 0}$ & $\mathbf{2 0 1 1}$ & $\mathbf{2 0 1 0}$ & $\mathbf{2 0 1 1}$ & $\mathbf{2 0 1 0}$ & $\mathbf{2 0 1 1}$ & $\mathbf{2 0 1 0}$ & $\mathbf{2 0 1 1}$ \\
\hline Control & 30.60 & 32.67 & 135.0 & 198.5 & 11.16 & 14.49 & 2.230 & 2.893 & 16.75 & 21.73 \\
\hline $\begin{array}{l}\text { Milagrow } \\
\text { (50000 ppm) }\end{array}$ & 39.71 & 40.72 & 147.0 & 292.0 & 15.53 & 31.61 & 3.130 & 6.320 & 31.35 & 63.22 \\
\hline Pepton (1000 ppm) & 33.00 & 33.77 & 142.0 & 202.0 & 11.95 & 23.41 & 2.383 & 4.677 & 23.91 & 79.02 \\
\hline Folgers (1000 ppm) & 57.40 & 57.46 & 169.0 & 350.5 & 17.27 & 37.99 & 3.450 & 7.660 & 34.55 & 76.65 \\
\hline $\begin{array}{l}\text { Gibberellins } \\
\text { (20 ppm) }\end{array}$ & 58.69 & 58.33 & 154.0 & 304.0 & 15.45 & 36.48 & 3.753 & 7.293 & 38.62 & 91.19 \\
\hline $\begin{array}{l}\text { Milagrow + } \\
\text { Pepton }\end{array}$ & 49.94 & 50.00 & 250.0 & 301.5 & 26.72 & 32.68 & 5.337 & 6.533 & 53.45 & 65.37 \\
\hline $\begin{array}{l}\text { Milagrow + } \\
\text { Gibberellins }\end{array}$ & 53.93 & 55.94 & 166.0 & 292.5 & 16.97 & 27.42 & 3.390 & 5.480 & 33.93 & 54.85 \\
\hline $\begin{array}{l}\text { Pepton + } \\
\text { Gibberellins }\end{array}$ & 47.45 & 62.20 & 243.0 & 333.0 & 29.72 & 40.51 & 5.940 & 8.097 & 74.31 & 101.3 \\
\hline $\begin{array}{l}\text { Milagrow + } \\
\text { Pepton + } \\
\text { Gibberellins }\end{array}$ & 52.75 & 53.65 & 224.5 & 302.7 & 27.62 & 31.65 & 5.520 & 6.323 & 69.05 & 63.30 \\
\hline \begin{tabular}{l} 
L.S.D at 0.050 \\
\hline
\end{tabular} & 2.26 & 3.61 & 3.1 & 3.4 & 1.79 & 1.95 & 1.271 & 1.217 & 1.47 & 1.14 \\
\hline
\end{tabular}

Meanwhile, the former studies cleared that, $\mathrm{GA}_{3}$ spray significantly increased the fruit set percentage of persimmon (Wally et al., 1999) pear (Kabeel \& Fawaaz, 2005, Chitu et al., 2007 and Nasr et al., 2009) and apricot (Hoseen et al., 2008). Also, Sugiyama and Kuraishi (1989) on "Navel" orange and Eissa et al. (2007) reported higher fruit set as a result of Milagrow (Brassinolide) and Folgers spray on Le-Conte pear. Also, Braun and Wild (1984), Kalinich et al.

Egypt. J. Hort. Vol. 40, No.2 (2013) 
(1985), Vardhini and Rao (1998) as well as Hayat et al. (2000) and Gabr et el. (2011) reported a significant increase in fruit yield/ tree as a result of Milagrow which may improves the assimilation efficiency of photosynthetic carbon and protein biosynthesis. El-Fakharany et al. (1995), Makarem and Mokhtar (1996) and Shahin et al. (2010) obtained the best fruit yield of " Anna " apple by $\mathrm{GA}_{3}$ spray at full bloom, fruit set and after 3 weeks. Eissa et al. (2007) improved pear yield components by Folgers spray. Abo Sedera et al. (2010) and Shehata et al. (2011) get better yield by the treatments of amino acids.

\section{Fruit characteristics}

Data in Tables 4, 5 \& 6 showed the response of "Costata" persimmon fruit characteristics (Fruit weight, size, dimensions, firmness and colour as well as fruit juice T.S.S., acidity and tannins) to the present treatments. Generally, GA, Pepton $+\mathrm{GA}_{3}$ and Pepton $+\mathrm{GA}_{3}+$ Milagrow induced the highest significant fruit weight and size in the two studied seasons. With regard to fruit firmness Millagrow (12.44 and $13.63 \mathrm{Ib} /$ inch $^{2}$ ) Pepton $+\mathrm{GA}_{3}$ (12.92 and $12.89 \mathrm{Ib} / \mathrm{inch}^{2}$ ) as well as Milagrow + Pepton $+\mathrm{GA}_{3}$ (12.04 and 12.20lb/inch ${ }^{2}$ ) supported persimmon fruit to be more suitable firm Moreover, the present treatments clearly improved the fruit colour at harvest specially Milagrow, $\mathrm{GA}_{3}$ and the combination treatments (Table 5). On the other hand, the fruit dimensions (length and diamenter) did not show a clear trend. However, the fruit juice T.S.S. increased significantly as affected by Milagrow, Pepton $+\mathrm{GA}_{3}$ and Milagrow + Pepton (21.0,19.5 and 19.0\%) in the $1^{\text {st }}$ season and by Milagrow + Pepton, Milagrow + Papton $+\mathrm{GA}_{3}$ and Milagrow $\left(20.0,20.0\right.$ and 19.0\%) in the $2^{\text {nd }}$ season, respectively (Table 6), The present treatments could significantly lower the fruit juice acidity than the control $(0.32$ and $0.26 \%)$. The most effective treatments in 2010 season were Folgers and Milagrow $+\mathrm{GA}_{3}(0.10$ and $0.10 \%)$ while in 2011season were Pepton $+\mathrm{GA}_{3}$, Milagrow, and Milagrow + Pepton + $\mathrm{GA}_{3},(0.17,0.18$ and $0.18 \%)$, respectively. Moreover, Milagrow + Pepton $+\mathrm{GA}_{3}$, Folgers and Milagrow + Pepton coused the least significant tannins in persimmon fruits through the two studied seasons while Milagrow significantly minimized the fruit juice Tannins only in the $2^{\text {nd }}$ season $(0.459 \%)$. The positive effect of these treatments extended to "Costata" "persimmon fruits after harvest. However, Table 7 showed that Pepton $+\mathrm{GA}_{3}$ and Milagrow + Peptone applications recorded the least decay percent after 21 days at room temperature (25 and 33.3\% respectively). Moreover, Milagrow + Pepton, $\mathrm{GA}_{3}$, Milagrow + $\mathrm{GA}_{3}$ and Peptone $+\mathrm{GA}_{3}$ treatments clearly stretched the fruit shelf life to 49 days in the cold storage (at $5^{\circ} \mathrm{C}$ ) than control $(71.4 \%$ ) or the other treatments where they effectively minimized the percentage of fruit decay to $37.5,42.9,42.9 \%$ and $42.9 \%$, respectively. 
TABLE 4. Effect of stimulant treatments on physical characteristics of "Costata" Persimmon fruits.

\begin{tabular}{|l|c|c|c|c|c|c|c|c|}
\hline \multirow{2}{*}{ Treatments } & \multicolumn{2}{|c|}{$\begin{array}{r}\text { Fruit weight } \\
(\mathbf{g})\end{array}$} & \multicolumn{2}{c|}{$\begin{array}{c}\text { Fruit size } \\
\left(\mathbf{c m}^{\mathbf{3}}\right)\end{array}$} & \multicolumn{2}{c|}{$\begin{array}{c}\text { Fruit length } \\
(\mathbf{c m})\end{array}$} & \multicolumn{2}{c|}{$\begin{array}{c}\text { Fruit diameter } \\
(\mathbf{c m})\end{array}$} \\
\cline { 2 - 10 } & $\mathbf{2 0 1 0}$ & $\mathbf{2 0 1 1}$ & $\mathbf{2 0 1 0}$ & $\mathbf{2 0 1 1}$ & $\mathbf{2 0 1 0}$ & $\mathbf{2 0 1 1}$ & $\mathbf{2 0 1 0}$ & $\mathbf{2 0 1 1}$ \\
\hline Control & 82.78 & 73.00 & 73.0 & 71.0 & 6.200 & 5.500 & 5.600 & 5.400 \\
\hline $\begin{array}{l}\text { Milagrow } \\
\text { (50000 ppm) }\end{array}$ & 106.7 & 108.3 & 109.0 & 109.0 & 5.467 & 5.400 & 6.000 & 5.967 \\
\hline $\begin{array}{l}\text { Pepton } \\
(1000 \text { ppm) }\end{array}$ & 106.8 & 115.9 & 95.0 & 110.0 & 6.033 & 5.700 & 6.000 & 5.467 \\
\hline $\begin{array}{l}\text { Folgers } \\
\text { (1000 ppm) }\end{array}$ & 101.9 & 109.4 & 101.0 & 111.0 & 5.467 & 5.933 & 5.400 & 5.433 \\
\hline $\begin{array}{l}\text { Gibberellins } \\
(20 \text { ppm) }\end{array}$ & 115.3 & 120.0 & 117.0 & 121.0 & 6.133 & 6.167 & 6.000 & 6.000 \\
\hline $\begin{array}{l}\text { Milagrow + } \\
\text { Pepton }\end{array}$ & 106.9 & 108.3 & 111.0 & 109.0 & 5.533 & 6.000 & 5.767 & 5.800 \\
\hline $\begin{array}{l}\text { Milagrow + } \\
\text { Gibberellins }\end{array}$ & 102.2 & 93.78 & 102.6 & 95.0 & 6.067 & 5.367 & 5.533 & 5.400 \\
\hline $\begin{array}{l}\text { Pepton + } \\
\text { Gibberellins }\end{array}$ & 122.4 & 121.7 & 126.0 & 121.0 & 6.067 & 6.367 & 5.967 & 6.167 \\
\hline $\begin{array}{l}\text { Milagrow + } \\
\text { Pepton + } \\
\text { Gibberellins }\end{array}$ & 123.1 & 104.4 & 122.0 & 108.0 & 6.267 & 6.100 & 5.967 & 5.800 \\
\hline L.S.D at 0.050 & 1.4 & 1.5 & 1.7 & 1.3 & 0.094 & 0.054 & 0.077 & 0.074 \\
\hline
\end{tabular}

TABLE 5. Effect of stimulant treatments on the fruit colour of "Costata" persimmon trees.

\begin{tabular}{|c|c|c|c|c|c|}
\hline Treatments & \multicolumn{3}{|c|}{2010} & \multicolumn{2}{|c|}{2011} \\
\hline Control & Majolica & yellow & $59 / 1$ & Orange buff & 507 \\
\hline $\begin{array}{l}\text { Milagrow } \\
(50000 \mathrm{ppm})\end{array}$ & Tangerine & orange & 8 & Tangerine orange & 9 \\
\hline Pepton (1000 ppm) & Majolica & yellow & $59 / 1$ & Spanish orange & $010 / 1$ \\
\hline Folgers $(1000 \mathrm{ppm})$ & Majolica & yellow & $59 / 1$ & Carrot read & $612 / 1$ \\
\hline Gibberellins (20 ppm) & Spanish & orange & 010/1 & Majolica yellow & $59 / 1$ \\
\hline Milagrow + Pepton & Cadmium & orange & 8 & Tangerine orange & 9 \\
\hline $\begin{array}{l}\text { Milagrow + } \\
\text { Gibberellins }\end{array}$ & Yellow & ocher & $07 / 1$ & Yellow & $07 / 1$ \\
\hline Pepton + Gibberellins & Cadmium & orange & 8 & Yellow & $07 / 1$ \\
\hline $\begin{array}{l}\text { Milagrow + Pepton + } \\
\text { Gibberellins }\end{array}$ & Yellow & ocher & $07 / 1$ & Tangerine orange & 9 \\
\hline
\end{tabular}

Egypt. J. Hort. Vol. 40, No.2 (2013) 
IMPROVING GROWTH, FRUIT SET, YIELD, FRUIT QUALITY...

TABLE 6. Effect of stimulant treatments on physical and chemical properties of "Costata" persimmon fruits .

\begin{tabular}{|l|c|c|c|c|c|c|c|c|}
\hline \multirow{2}{*}{\multicolumn{1}{|c|}{ Treatments }} & \multicolumn{2}{|c|}{$\begin{array}{c}\text { Fruit Firmness } \\
\left(\mathbf{L b} / \mathbf{i n c h}{ }^{2}\right)\end{array}$} & \multicolumn{2}{c|}{ T.S.S.\% } & \multicolumn{2}{c|}{ Acidity \% } & \multicolumn{2}{c|}{ Tannin \% } \\
\cline { 2 - 10 } & $\mathbf{2 0 1 0}$ & $\mathbf{2 0 1 1}$ & $\mathbf{2 0 1 0}$ & $\mathbf{2 0 1 1}$ & $\mathbf{2 0 1 0}$ & $\mathbf{2 0 1 1}$ & $\mathbf{2 0 1 0}$ & $\mathbf{2 0 1 1}$ \\
\hline Control & 11.27 & 9.50 & 16.50 & 17.50 & 0.32 & 0.26 & 1.742 & 1.366 \\
\hline $\begin{array}{l}\text { Milagrow } \\
\text { (50000 ppm) }\end{array}$ & 12.44 & 13.63 & 21.00 & 19.00 & 0.34 & 0.18 & 1.330 & 0.459 \\
\hline Pepton (1000 ppm) & 8.817 & 9.160 & 11.50 & 16.50 & 0.18 & 0.28 & 1.454 & 1.454 \\
\hline $\begin{array}{l}\text { Folgers } \\
\text { (1000 ppm) }\end{array}$ & 6.230 & 10.92 & 13.50 & 13.00 & 0.10 & 0.22 & 0.365 & 0.499 \\
\hline $\begin{array}{l}\text { Gibberellins } \\
\text { (20 ppm) }\end{array}$ & 8.56 & 10.86 & 13.00 & 15.50 & 0.16 & 0.22 & 0.998 & 0.998 \\
\hline Milagrow + Pepton & 11.23 & 11.03 & 19.00 & 20.00 & 0.20 & 0.32 & 0.374 & 0.374 \\
\hline $\begin{array}{l}\text { Milagrow + } \\
\text { Gibberellins }\end{array}$ & 10.22 & 10.68 & 16.00 & 16.00 & 0.10 & 0.20 & 1.945 & 1.790 \\
\hline $\begin{array}{l}\text { Pepton + } \\
\text { Gibberellins }\end{array}$ & 12.92 & 12.89 & 19.50 & 18.50 & 0.17 & 0.17 & 0.665 & 0.665 \\
\hline $\begin{array}{l}\text { Milagrow + Pepton } \\
\text { + Gibberellins }\end{array}$ & 12.04 & 12.20 & 12.00 & 20.00 & 0.16 & 0.18 & 0.208 & 0.208 \\
\hline L.S.D at 0.050 & 0.27 & 0.39 & 1.48 & 0.99 & 0.06 & 0.05 & 0.145 & 0.155 \\
\hline
\end{tabular}

Generally, the present findings are supported by the former investigators where Wally et al. (1999) on persimmon and Eissa et al. (2007) on pear reported a significant increase in fruit quality as a result of Folgers spray. Also Refaat and Naguib (1998) as well as Abo-Sedera et al. (2010) and Ibrahim et al. (2010) get an increase of fruit weight and T.S.S. as a result of Pepton (amino acids) spray which may have important role on the biosynthesis of chlorophyll molecules and in turn affected carbohydrate content. In addition to, Milagrow (Brassinolides) application increased fruit weight and T.S.S. while decreased fruit juice acidity of orange (Wang et al., 2004) grape, (Symons et al., 2006) and apricot (Gabr et al., 2011) as well as promoted fruit ripening.

Meanwhile, Eliwa et al. (1998) on persimmon and Hoseen et al. (2008) on apricot did not obtain clear effect of $\mathrm{GA}_{3}$ application on fruit quality. Contrarily, Nasr et al. (2009) and Wally and Abdellatif (2009), showed that $\mathrm{GA}_{3}$ spray improved apricot fruit quality. Moreover, Fruit juice Tannins of "costata" persimmon did not affected by $\mathrm{GA}_{3}$ spray as Eliwa et al. (1998) and Wally et al. (1999). Differently, Guirguis et al. (2009) stated that, $\mathrm{GA}_{3}$ treatment lowered tannins content in "Mackawa Jiro" persimmon subsequently affected its harvest date as well as increased its shelf-life period and storage ability. 


\begin{tabular}{|c|c|c|c|c|c|c|c|c|c|c|}
\hline \multirow{2}{*}{ 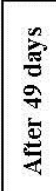 } & 힝 & $\stackrel{F}{*}$ & ڤ్ర & $\underset{\sim}{\stackrel{*}{r}}$ & $\vec{i}$ & वे & $\frac{n}{n}$ & $\stackrel{\text { qे }}{\text { qे }}$ & ఫे & in \\
\hline & $\begin{array}{l}\bar{\Xi} \\
\check{q}\end{array}$ & 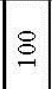 & 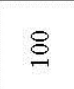 & 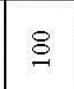 & 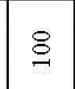 & 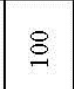 & 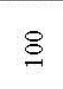 & 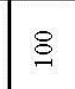 & 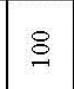 & $\S$ \\
\hline \multirow{2}{*}{ 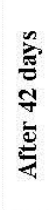 } & 들 & $\vec{\square}$ & $\stackrel{\circ}{\circ}$ & $\overrightarrow{\vec{n}}$ & 0 & $\stackrel{m}{ \pm}$ & $\stackrel{\leftrightarrow}{\dot{C}}$ & $\circ$ & 0 & 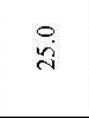 \\
\hline & 言 & $\stackrel{8}{\circ}$ & 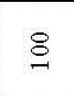 & $\stackrel{\&}{-}$ & $\stackrel{8}{-}$ & $\stackrel{\&}{\stackrel{-}{1}}$ & 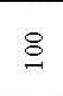 & $\stackrel{\&}{-}$ & $\stackrel{8}{9}$ & $\stackrel{\S}{\leftrightarrows}$ \\
\hline \multirow{2}{*}{ 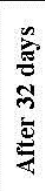 } & 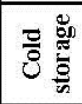 & $\begin{array}{l}0 \\
\stackrel{\infty}{d} \\
\end{array}$ & $\stackrel{m}{ \pm}$ & $\dot{\text { ì }}$ & 0 & $\stackrel{m}{ \pm}$ & $\stackrel{\circ}{\dot{C}}$ & 0 & 0 & $\stackrel{8}{\sim}$ \\
\hline & 言 & 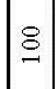 & $\stackrel{8}{\circ}$ & $\stackrel{\S}{-}$ & $\stackrel{8}{=}$ & $\stackrel{\&}{\circ}$ & $\stackrel{8}{9}$ & $\stackrel{8}{\circledR}$ & $\stackrel{8}{\circ}$ & $\S$ \\
\hline \multirow{2}{*}{ 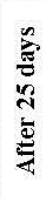 } & 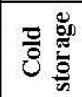 & $\stackrel{?}{ \pm}$ & $\circ$ & $\stackrel{m}{\exists}$ & 0 & $\stackrel{?}{\exists}$ & $\stackrel{?}{\exists}$ & 0 & 0 & $\stackrel{\sim}{\underline{y}}$ \\
\hline & 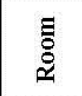 & $\stackrel{8}{\circ}$ & 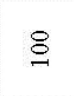 & $\stackrel{\&}{-}$ & $\stackrel{\&}{-}$ & $\stackrel{\&}{\leftrightarrows}$ & 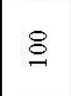 & $\stackrel{8}{9}$ & 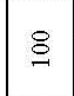 & 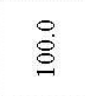 \\
\hline \multirow{2}{*}{ 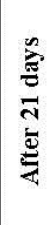 } & 들 & 0 & 0 & 0 & $\circ$ & 0 & 0 & $\circ$ & 0 & 0 \\
\hline & $\begin{array}{l}\text { 言 } \\
\text { व }\end{array}$ & $\stackrel{\stackrel{8}{8}}{\circ}$ & $\stackrel{8}{\stackrel{8}{8}}$ & $\stackrel{\ominus}{\S}$ & 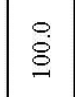 & $\begin{array}{l}0 \\
:\end{array}$ & $m_{m}^{m}$ & $\stackrel{\stackrel{\Xi}{\Xi}}{\circ}$ & i. & $\stackrel{\circ}{\mathrm{r}}$ \\
\hline \multirow{2}{*}{ 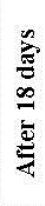 } & 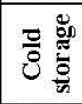 & 0 & o & 0 & 0 & 0 & o & 0 & $\circ$ & 0 \\
\hline & 言 & $\stackrel{\leftrightarrow}{\dot{\leftrightarrow}}$ & $\stackrel{8}{\stackrel{\Xi}{\leftrightarrows}}$ & $\stackrel{\stackrel{i}{r}}{r}$ & $\stackrel{\leftrightarrow}{\stackrel{\leftrightarrow}{~}}$ & $\stackrel{\circ}{\circ}$ & $\stackrel{\leftrightarrow}{\stackrel{\sim}{~}}$ & : & ì & $\stackrel{\sim}{\circ}$ \\
\hline \multirow{2}{*}{ 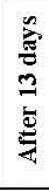 } & 일 & 0 & 0 & 0 & 0 & o & 0 & $\circ$ & 0 & 0 \\
\hline & 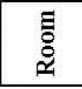 & 0 & 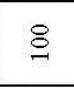 & $\stackrel{?}{ \pm}$ & 0 & $\stackrel{\sim}{\mathrm{i}}$ & 0 & $\stackrel{\leftrightarrow}{\mathrm{i}}$ & $\circ$ & 0 \\
\hline \multicolumn{2}{|c|}{ 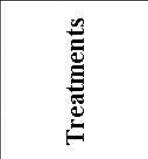 } & $\stackrel{0}{=}$ & 总哭递 & 言 & 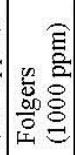 & 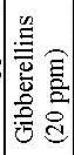 & 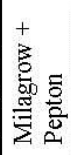 & 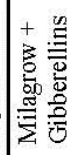 & 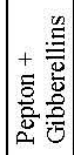 & 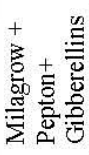 \\
\hline
\end{tabular}


The present data showed a positive effect (mostly significant) of the studied treatments (especially GA3 and Folgers treatments) on the leaf area and number of leaves (Table 1). The increase of number of leaves and leaf area mean much dense photosynthesis. However, data illustrated in Fig. 1, 2, 3 indicate a positive correlation and regression between leaf area (Independent factor) as well as all of fruit set percentage, fruit yield and juice TSS (Dependent Factors) through the two studied seasons.
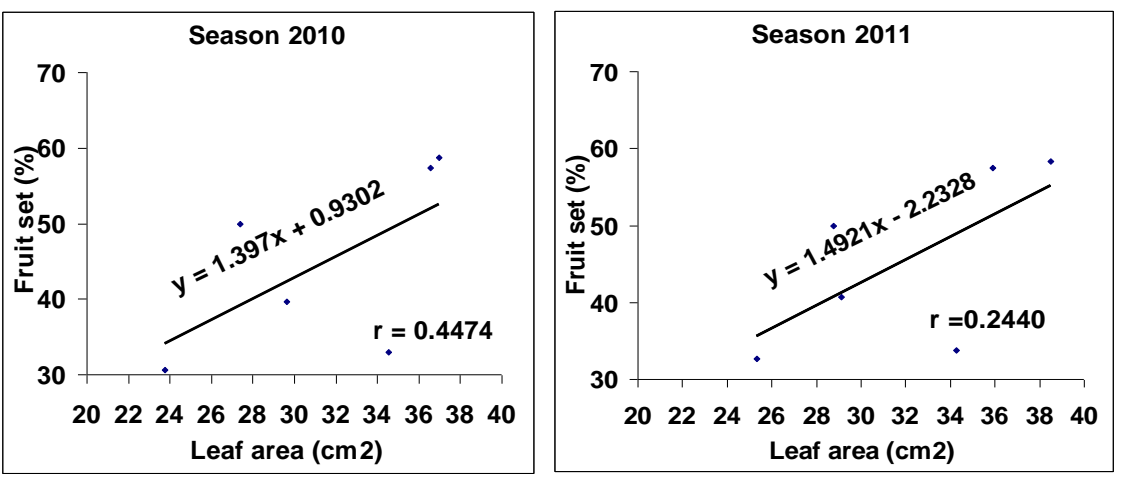

Fig.1. Relationship between leaf area $\left(\mathrm{cm}^{2}\right)$ and fruit set $(\%)$.
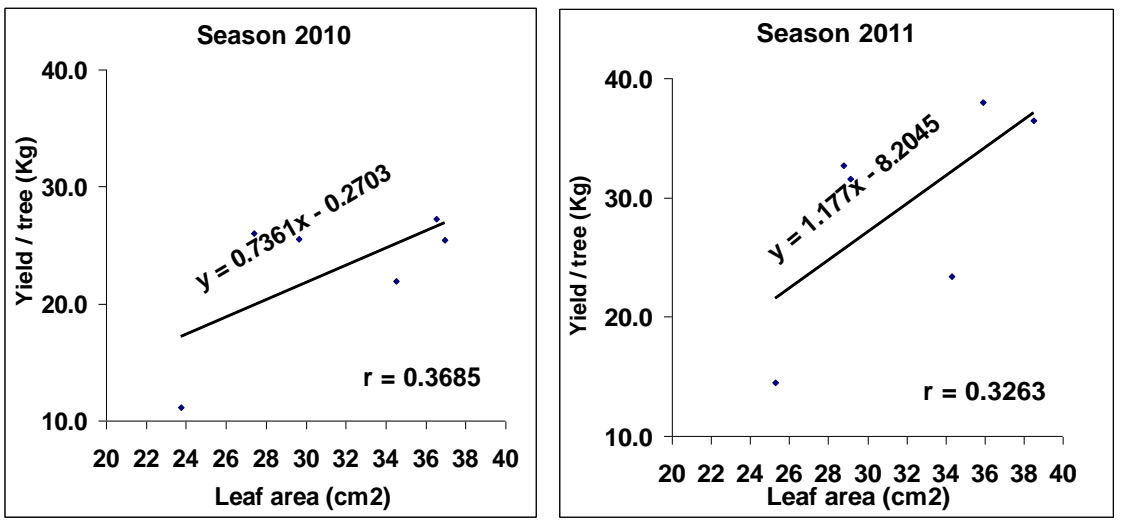

Fig. 2. Relationship between leaf area $\left(\mathrm{cm}^{2}\right)$ and yield / tree $(\mathrm{Kg})$. 

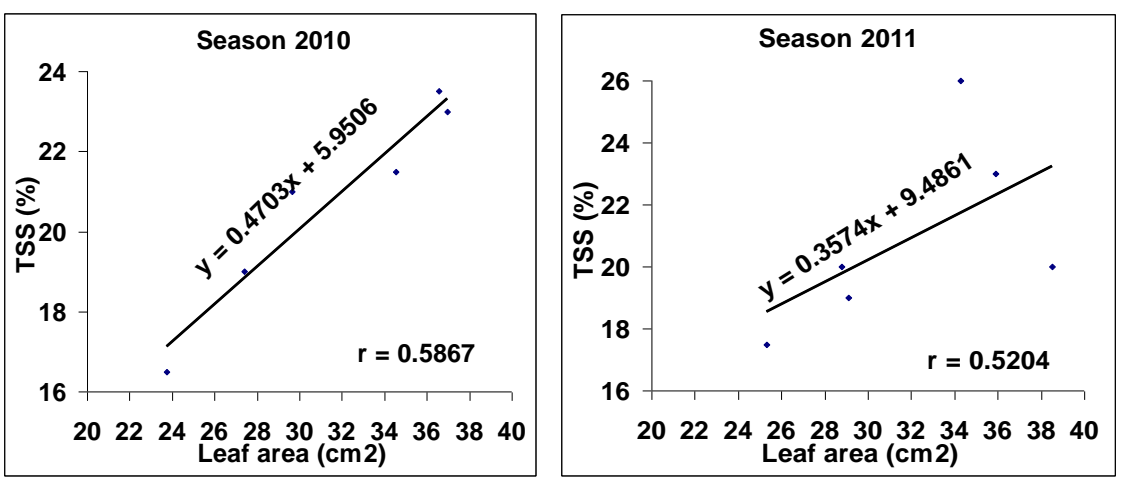

Fig. 3. Relationship between leaf area $\left(\mathrm{cm}^{2}\right)$ and TSS (\%).

\section{Conclusions}

The present data showed that, the most treatments positively affected "Costata" persimmon growth. However, we can conclude that, single treatments were more effective on vegetative growth. Also, Folgers, $\mathrm{GA}_{3}$ and Milagrow+ Pepton induced significant increase in leaf $\mathrm{N}$ and $\mathrm{K}$ while leaf $\mathrm{P}$ content did not show clear trend. Meanwhile, single and combined treatments caused obvious increase in yield components and fruit quality attributes. Therefore, we can recommend the growers of "Castata" persimmon to spray their orchards three times: at full bloom, fruit set and at fruit drop with Pepton $+\mathrm{GA}_{3}$ which induced the highest fruit set percentage, number of fruits / tree, fruit yield with more suitable firmness and longer shelf life as well as much monetary value to increase their income.

\section{References}

Abd El-Aziz, N.G. and Balbaa, L. (2007) Influence of tyrosine and zinc on growth, flowering and chemical constituents of saliva farinaceae plants. Egypt. J. Appl. Sci., 3 (11), $1479-1489$.

Abd El- Ghany, N.A. (2005) Fruit drop. Agricultural J., 60 (3), 21-22. ministry of agric. Egypt.

Abo Sedera, F.A., Abd El-Latif, A., Bader, L.A.A. and Rezk, S.M. (2010) Effect of NPK mineral fertilizer levels and foliar application with humic and amino acids on yield and quality of strawberry. Egypt. J. of Appl. Sci., 25 , 154-169.

Aly, M.A., Ezz, T.M., Abd El-Megeed, N.A. and Naseb, F.A. (2012) Improving " Leconte" pear trees productivity by foliar application with plant bioregulators and boric acid. J. Adv. Agric. Res. (Fac. Agric. Saba Basha) 17 (3) , 622-643.

A.O.A.C. (1970) Official Method of Anlaysis, Association of official analytical chemists, Washington, D. C., $11^{\text {th }}$ ed., p. 240.

Egypt. J. Hort. Vol. 40, No.2 (2013) 
Awad, N. and Amenomori, H. (1971) The effect of gibberellin on the ripening of persimmon Diapason Kaki, Cv. Tabate. Revista de Agriculture, piraciacaba , Brazil. (Host. Abst. 42: 6737).

Baghdady, H.A. and Mineasy, F. (1964) The Fruit Production. Text book, Dar ElMaarif, Egypt. p. 563.

Bleasdale, L.K.A. (1978) Plant physiology in relation to horticulture. ELBS. edition. The Macmillan press LTD. Associated companies in Delhi, Hong Kong Lagos and Singapore.

Braun, P. and Wild, A. (1984) The influence of brassinosteroid on growth and parameters of wheat and mustard plants. J. Physical., 49, $427-451$.

Chitu, V., Chitu, E. and Braniste, N. (2007) Effects of $\mathrm{GA}_{3}$ and paclobutrazol treatments on fruit set and yield "Beurre Bose" and "Triumf" pear cultivars. Acta Host., 800, $452-465$.

Clause, S.D. (1996) Molecular genetic studies confirm the role of brassionstesoids in plant growth and development. Plant J.,10 (1), 1-8.

Datir, R.B., Apparao, B.J. and Laware, S.L. (2012) Application of amino acid chelated micronutrients for enhancing growth and productivity in chili (Capricum annum L. ). Plant sci. feed, 7, $100-105$.

Eissa, F.M., Fathi, M.A. and Kandil, E.A. (2007) Response of "Le-Conte" pear (Pyrus communis L.) trees to foliar application with some biostimulants. Minufiya. J. Agric. Res., 32 (4), $1143-1154$.

El-Fakharany, M.M., Wally, A.S. and Makarim, M.M. (1995) Effect of some regulators on " Anna" apple retained fruits and fruit quality. J. Agric. Sc. Mansoura Univ., 20 (10), 4439 -4446.

El-Fakharani, E.M.M., Wally, A.S. and Saied, I.A. (1990) Effect of some growth regulators on persimmon. 1-Effect of Alar and $\mathrm{GA}_{3}$ on retained fruits and fruit quality of persimmon. Annals, Agric. Sci. Moshtohor, 28 (3), $1699-1710$.

Eliwa, G.I., Wally, A.S.M. and Ali, M.M. (1998) Effect of calyx sepal removal and $\mathrm{GA}_{3}$ spraying on "Costata" persimmon fruit characteristics. J. Agric. Sci. Mansoura Univ., $23(2), 831-841$.

Gabr, M.A., Fathi M.A., Mohamed, A.I. and Mckhaeil, G.B. (2011) Influences of some chemical substances used to induce early harvest of ' Canino ' apricot trees. Nature and Science, 9 (8), 59 - 65.

Gomes, M.M.A., Campostini, E., Leal, N.R., Viana, A.P., Ferraz, T.M., Siqueira, L. N., Rosa, R.C.C., Netto, A.T., Nunez-Vazquez, M. and Zullo, M.A.T. (2006) Brassinosteroid analogue effect on the yield of yellow passion fruit plants, Scientia Horti., 110, 235 - 240. 
Gross, J., Baza, H., Blumenfeld, A. and Ben - Ari, R. (1984) Changes in chlorophyll and carotenoid pigments in the peel of "Triumph" persimmon (Diospyres Kaki L. ) induced by pre-harvest gibberellin $\left(\mathrm{GA}_{3}\right)$ treatments. Scienta Horticulture , 24 (3/4), $305-314$. (Host . Abst. , $55: 3936$ ).

Guirguis, N.S., Nabil, W.A. and Nasr, M. (2009) Effect of sitofex and $\mathrm{GA}_{3}$ spray and time of application on fruit set, yield and fruit quality of " Mackawa Jiro " Kaki cultivar. Annales of Agric. Sci, Moshtohor, 47 (1), 29-39.

Gul, H. J., Khattak, A. M. and Amin, N. (2006) Accelerating the growth of Araucaria Heterophylla seedlings throught different gibberellic acid concentrations and nitrogen levels. J. Agric. Boil. Sci., 1, 25-29.

Hasegawa, K., Kuge, N., Minura, T. and Nakajima, Y. (1991) Effects of KT-30 and $\mathrm{GA}_{3}$ on the fruit set and fruit growth of persimmon CVS. J. Japanese Soc. Hort. Sci., 60 (1), 19-29.

Hassan, H.S.A., Sarrwy, S.M.A. and Mostafa, E.A.M. (2010) Effect of foliar spraying with liquid organic fertilizer, some micronutrients and gibberellins on leaf mineral content, fruit set, yield, and fruit quality of " Hollywood " plum trees, Agric. and Bio. J. Nor. Amer., 2151-7525.

Hayat, S., Ahmad, A., Mobin, M., Hussain, A. and Fariduddin, Q. (2000) Photosynthetic rate, growth and yield of mustard plants splayed with 28homobrassinolide. Photosynthetica, 38, 469-471.

Hoseen, S., Abou Grah, F.I.I. and Wally, A.S. (2008) Effect of foliar spraying of gibberellic acid after harvest on apricot "Canino" fruit yield and quality, J. Agric. Sci. Mansoura Univ., 33 (2), 1365 - 1374.

Ibrahim, S.M., Taha, L.S. and Farahat, M.M. (2010) Influence of foliar application of Peptone on growth, flowering and chemical composition of Helichrysum bracteatum plants under different irrigation intervals. Ozean J. Appl. Sci., 3 (1), $1943-2429$.

Kabeel, H. and Fawaaz, S.A.A. (2005) Effect of spraying some growth regulators on " Le conte " pear trees on : I. productivity, fruit quality and leaf mineral content. Res. J. Soc. En. Sci., 3 (3), 172- 193

Kalinich, J.F., Mandava, N.B. and Todhunter, J.A. (1985) Relationship of nucleic acid metabolism to brassinolide - induced responses in beans. Plant Physiol, 120, 207-214.

Kauschmann, A., Jessop, A., Konc, C., Szekeres, M., Willmitzer, L. and ALtmann, T. (1996) Genetic evidence for an essential role of brassinosteroids in plant development. The Plant J., 9 (5), 701- 713.

Kowalczyk, K. and Zielony, T. (2008) Effect of Amino acids and Asahi on yield and quality of lettuce grown on rockwool. Conf. of biostimulators in modern Agriculture, 7- 8 Feb. , Warsaw, Poland.

Krizek, D.T. and Mandava, N.B. (1983) Influence of spectral quality on the growth response of intact bean plants to brassinosteriod, a growth - promoting steroidal lactone. I. Stem elongation and morphogenesis. Physiologia Plantarum , 57 (3), 317- 323.

Egypt. J. Hort. Vol. 40, No.2 (2013) 
Looney, N.E. (1993) Improving fruit size, appearance and other aspects of fruit crop " quality" with plant bioregulating chemicals. Acta-Horti., 329 , 120-127.

Makarem, M.M. and Mokhtar, H. (1996) Effect of biozyme and $\mathrm{GA}_{3}$ on fruit set, yield and fruit quality of " Anna" apple trees. J. Agric. Sci. Mansoura Univ., 21 (7), 26612669.

Mokhtar, H. and Wally, A.S.M. (1999) Effect of defoliation date on persimmon " costata" vegetative and flower bud development, fruit set and characteristics. Egypt. J. Agric. Res., 77 (1), 289 - 303.

Montoya, T., Nomura, T., Yokota, T., Farrar, K., Harrison, K., Jones, J., Kaneta, T., Kamiya, Y., Szekeres, M. and Bishop, G. (2005) Patterns of dwarf expression and brassinosteriod accumulation in tomato reveal the importance of brassinosteriod synthesis during fruit development. The plant J., 42 (2), $262-269$.

Mussig, C. (2005) Brassionsteroid - promoted growth. Plant Biol., 2, 110 -117.

Myers C.S. and Ferree, D.C. (1983) Influence of summer purring and tree orientation on net photosynthesis transpiration, shoot growth, and dry- weight distribution in young apple trees . J. Amer-Soc. Host . Sci., 108 (1), 4-9.

Nasr, M.M., Mohamed, S.A. and Gehad, B.M. (2009) Effect of some compounds on fruit yield, fruit quality and storability of " Le- Conte " pear., J. Biol. Chem. Environ. Sci., 4 (1), $985-1012$.

Refaat, A.M. and Naguib, N.Y. (1998) Peppermint yield and oil quality as affected by application of some amino acids. Bull. Fac. Agric. Univ. Cairo, 49, 89 - 98.

Russo, R.O. and Berlyn, G.P. (1990) The use of organic biostimoulants to help low input sustainable agriculture. J. Sustainable Agric., 1 (2), 19 - 42.

Shahin, M.F.M., Fawzi, M.I.F. and Kandil, E.A. (2010) Influence of foliar application of some nutrient (fertifol Misr) and $\mathrm{GA}_{3}$ on fruit set, yield, fruit quality and leaf composition of "Anna" apple trees growth in sandy soil , J. Amer. Sci., 6 (12) , 202-208.

Shehata, S.M., Abdel-Azem, H.S., Abou El-Yazied, A. and El-Gizawy, A.M. (2011) Effect of foliar spraying with amino acids and seaweed extract on growth chemical constitutes, yield and its quality of Celeriac plant. European J. of Scientific Research, 58 (2), 257- 265.

Shirzad, S., Hosein, A. and Majid, A. (2013) Effect of $\mathrm{GA}^{3}$ and Ehtephon on sex expression and oil yield in medicinal pumpkin (Cucurbita peop var. styriaca). Intl. J. Farm. \& Alli. Sci., 2 (9), 196-201.

Snedecor, G.W. and Cochran, W.G. (1990) Statistical Methods. $7^{\text {th }}$ ed. The Iowa State Univ. press. Ames Iowa, USA. p. 593.

Sugiyama, K. and Kuraishi, S. (1989) Stimulation of fruit set of "Morita" navel orange with brassinolide VI : international symposium on growth regulators in fruit production. Acta Horti ., 239 , 345 - 348 . 
Symons, G.M., Davies, C., Shavrukov, Y., Dry, I.B., Reid, J.B. and Thomas, M.R. (2006) Grapes in steroids. Brassionsteroid are involved in grape berry ripening. Plant Physiol., 140 (1), 150-158.

Thon, M., Maretzki, A., Komer, E. and Sokai, W.S. (1981) Nutrient uptake and accumulation by sugar - cane cell culture in relation to growth cycle. Plant Cell Tissue and Organ. Culture., 1, 3-14.

Vardhini, B. V. and Rao, S. S. R. (1998). Effect of brassinosteriods on growth, metabolit content and yield of Arachis hypogaea. Phytochemistesry, 48, $927-930$.

Wally, A.S. and Abdellatif, G.S. (2009) Effect of foliar spray with $\mathrm{GA}_{3}$ alone or combined with some fertilizers on yield and fruit quality of "Canino " apricot . Egypt. J. Apple . Agric. Res., 2 (2), 153- 163.

Wally, A.S.M., Mokhtar, H. and El-Fakharanay, E.M. (1999) Effect of biozyme and $\mathrm{GA}_{3}$ on fruit set, yield and fruit characters of " Costata " persimmon. Egypt. J. Agric. Res., 77 (2), $805-815$.

Walter, G.R. and Nawacki, E. (1978) Alkaolid Biolog and Metabolism in Plants. Planum press, N. Y., p. 152.

Wang, C.F., You, Y., Chen, F.L.X., Wang, J. and Wang, J.C. (2004) Adjusting effect of brssinolide and $\mathrm{GA}_{4}$ on the orange growth. Acta Agri. Jiangxiensis Univ., 5-22. 
IMPROVING GROWTH, FRUIT SET, YIELD, FRUIT QUALITY...

تحسين النمو والعقد والمحصول ومواصفات جودة الثمار وقابليتها للتخزين فى ثمار الكاكي صنف كوستاتا

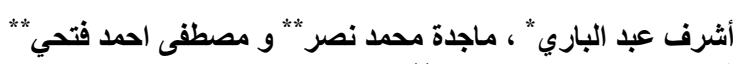

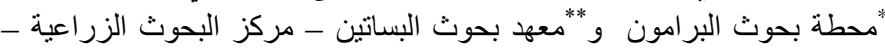

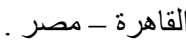

\footnotetext{
أجري هذا البحث فى مزرعة محطة البحوث الزراعية - البرامون - محافظة

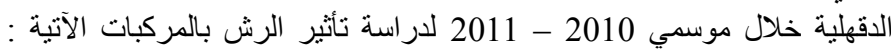

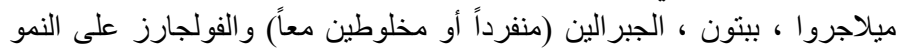

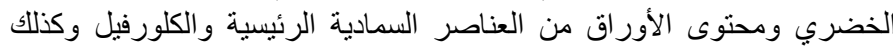

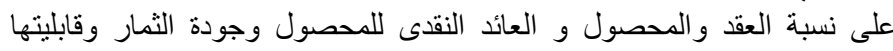

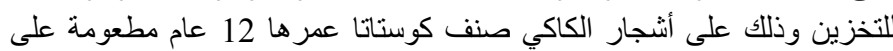
أصل بذري ونامية فى أرض طميية.

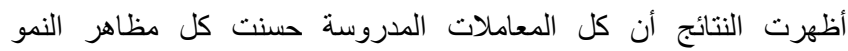

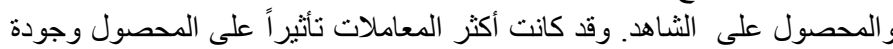

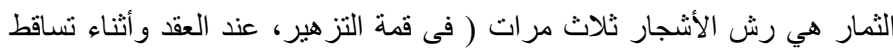

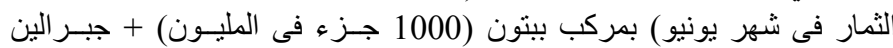

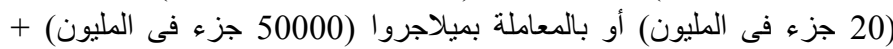

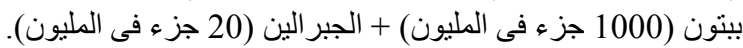

